\title{
Evaluación de los parámetros físicos y químicos del agua de mar en los alrededores de la Isla Uvita, Limón, Costa Rica
}

\section{Evaluation of physical and chemical parameters of sea water in the surroundings of Uvita Island, Limon, Costa Rica}

Jaime Alfaro-Sandí1, Gilberto Piedra-Marín², Ana Y. Saravia-Arguedas ${ }^{3}$, Lilliana Piedra-Castro ${ }^{4}$

Fecha de recepción: 5 de febrero de 2020

Fecha de aprobación: 14 de junio de 2020

Alfaro-Sandí, J; Piedra-Marín, G; Saravia-Arguedas, A.Y; Piedra-Castro, L. Evaluación de los parámetros físicos y químicos del agua de mar en los alrededores de la Isla Uvita, Limón, Costa Rica. Tecnología en Marcha. Vol. 34-2. AbrilJunio 2021. Pág 88-95.

doi) https://doi.org/10.18845/tm.v34i2.4985

1 Licenciado en Química Industrial. Jefe del Laboratorio de análisis de residuos de agroquímicos. Servicio Fitosanitario del Estado. Costa Rica. Correo electrónico: jasm138275@hotmail.com (D) https://orcid.org/0000-0002-7296-9895

2 Doctor en Química.Sección de Química Inorgánica, Escuela de Química, Universidad Nacional, Costa Rica.

Correo electrónico: gilberto.piedra.marin@una.ac.cr (iD https://orcid.org/0000-0003-1332-236X

3 Máster en Química. Laboratorio de Química Marina, Escuela de Química, Universidad Nacional, Costa Rica.

Correo electrónico: ana.saravia.arguedas@una.ac.cr (iD https://orcid.org/0000-0002-7296-9895

4 Doctora en Ciencias Naturales para el Desarrollo . Laboratorio de Recursos Naturales y Vida Silvestre, Escuela de Ciencias Biológicas, Universidad Nacional. Costa Rica. Correo electrónico: lilliana.piedra.castro@una.ac.cr 


\section{Palabras clave}

Emisario submarino; isla Uvita, Limón; contaminación; descargas.

\section{Resumen}

El presente trabajo evaluó una serie de parámetros físicos y químicos para determinar la calidad del agua marina en los alrededores de la isla Uvita, Limón, Costa Rica, y determinar la posible influencia de las descargas procedentes del emisario submarino de la ciudad de Limón sobre el entorno marino circundante. Se evaluaron nutrientes (nitritos, nitratos, silicatos, fosfatos y amonio), metales pesados (plomo, cromo, níquel), sólidos (disueltos y sedimentables) y parámetros físicos ( $\mathrm{pH}$, temperatura, turbiedad, etc.). La zona de estudio se dividió en segmentos circundantes a la isla y se ubicaron 6 puntos de muestreo alrededor de la misma con el fin de comparar la calidad del agua de las áreas con mayor influencia del emisario con las de menor influencia. Se programaron tres actividades de muestreo: setiembre 2012 (época Iluviosa), octubre 2012 (transición Iluviosa-seca) y setiembre 2013 (época lluviosa). Los niveles de nutrientes, metales pesados, sólidos y parámetros físicos, se compararon con los reportados en la literatura para el Golfo de México y el Mar Caribe, encontrándose que, en general, eran mayores a los reportados en la literatura. En particular, los datos del último muestreo mostraron un incremento considerable en la concentración de los metales pesados ( $\mathrm{Pb}, \mathrm{Cr}$ y $\mathrm{Ni}$ ), que podría ser atribuido a factores ajenos a las descargas del emisario como por ejemplo las operaciones portuarias y las actividades antropogénicas industriales, las cuales repercuten en la zona mencionada.

\section{Keywords}

Submarine emissary; Uvita island, Limón; pollution; discharges.

\section{Abstract}

The present work evaluated a series of physical and chemical parameters to determine the quality of marine water in the surroundings of Uvita island, Limón, Costa Rica, and to determine the possible influence of the discharges coming from the submarine emissary of the city of Limón on the surrounding marine environment. Nutrients (nitrites, nitrates, silicates, phosphates and ammonium), heavy metals (lead, chromium, nickel), solids (dissolved and sedimentable) and physical parameters ( $\mathrm{pH}$, temperature, turbidity, etc.) were evaluated. The study area was divided into segments surrounding Uvita island, and 6 sampling points were located around the island in order to compare the water quality of the areas with greater emissary influence with those of less influence. Three sampling activities were scheduled: September 2012 (rainy season), october 2012 (rainy-dry transition) and september 2013 (rainy season). The levels of nutrients, heavy metals, solids and physical parameters were compared with those reported in the literature for the Gulf of Mexico and the Caribbean Sea, finding that, in general, they were higher than those reported in the literature. In particular, the last sampling data showed a considerable increase in the concentration of the heavy metals ( $\mathrm{Pb}, \mathrm{Cr}$ and $\mathrm{Ni}$ ), which could be attributed to other factors outside the emissary's discharges such as port operations and industrial anthropogenic activities, which impact on the aforementioned area. 


\section{Introducción}

El manejo inadecuado de los recursos naturales en Costa Rica, en conjunto con las formas ineficientes de tratar los residuos sólidos y líquidos, se ha incrementado considerablemente en los últimos años; lo anterior obedece primordialmente a un crecimiento desproporcionado en los sectores económico, social e industrial, lo cual repercute en la contaminación y la degradación del medio ambiente, afectando directa o indirectamente las cuencas hidrográficas del país [1].

El deterioro de los cuerpos de agua superficiales aumenta cuando los sectores sociales e industriales disponen de manera inadecuada los desechos generados en las actividades cotidianas, siendo por lo general los vertederos finales los ríos, los humedales, los lagos, las lagunas, los mares y los océanos.

El auge y la tecnificación masiva de sectores como la industria de los alimentos, los servicios, la agricultura, la ganadería y el turismo, tienden a incrementar la generación de desechos de toda índole, entre los cuales destacan residuos tóxicos como metales pesados, plaguicidas y desechos electrónicos, entre otros. [1].

La disposición de aguas residuales en los cuerpos superficiales en Costa Rica es un problema que se ha intensificado debido a la carencia de sistemas eficientes de alcantarillado sanitario. Solo el $24,8 \%$ de la población costarricense está cubierto por el sistema de alcantarillado público; adicionalmente, los pocos existentes han colapsado debido al incremento de la población y a la ausencia del mantenimiento [2]. Se ha reportado que uno de cada 15 habitantes (6,6\% de la población) recibe el servicio de alcantarillado sanitario, siendo San José la provincia con la mayor cobertura (51\%), seguida por Heredia y Cartago (15\%), en las restantes provincias la cobertura es inferior al 10\% [3]

No todas las aguas colectadas por el sistema de alcantarillado son llevadas a una planta depuradora, la descarga de aguas residuales industriales y domésticas en los cuerpos de agua incrementa la cantidad de materia orgánica presente, lo que conlleva a una disminución del contenido de oxígeno disuelto que a su vez afecta los ecosistemas acuáticos. Asimismo, el incremento de los sólidos sedimentables provenientes de la sedimentación de la materia orgánica y la erosión del suelo ocasiona a mediano plazo, que un cuerpo de agua pierda la capacidad de soportar la diversidad acuática [3].

Las aguas superficiales que desembocan en las costas se ven afectadas por la presencia de microorganismos, compuestos inorgánicos, nutrientes, sólidos en suspensión, metales pesados, compuestos orgánicos (denominados "materia orgánica disuelta") e hidrocarburos, entre otros. En consecuencia, la calidad del agua dependerá del valor de los distintos parámetros físicos, químicos, bióticos y microbiológicos [3].

Una alternativa para disponer los desechos que tienen por destino final los océanos es el uso de sistemas de difusión, entre los que destacan los emisarios submarinos. La ciudad de Limón no escapa a la problemática de la generación de residuos líquidos y sólidos; por ello, en el año 2005 se instaló un emisario submarino para el tratamiento de los residuos líquidos. Este dispositivo consiste en un ducto cerrado que transporta las aguas negras urbanas, de origen doméstico o industrial, desde una planta de tratamiento preliminar hasta mar abierto, a cierta distancia de la costa (2 km mar adentro), con la intención de prevenir la contaminación en los alrededores de la zona costera [1]. El emisario submarino de la ciudad de Limón se encuentra ubicado entre la isla y la costa, a menos de $1 \mathrm{~km}$ del arrecife isleño, como se muestra en la figura 1. Las aguas de la planta de tratamiento se bombean hacia los difusores del emisario submarino formando una pluma que simultáneamente se desplaza por debajo de la termoclina y se diluye en las aguas marinas, evitando que los efluentes lleguen a la superficie y puedan eutrofizar el agua circundante [4]. 
Estudios de impacto ambiental evaluados en los últimos 15 años por la Secretaría Técnica Nacional Ambiental mostraron que los efectos de contaminantes como hidrocarburos, metales pesados, nutrientes y materia orgánica presentes en las aguas de vertido industrial y domésticas pueden causar una disminución en la flora y la fauna características de los ecosistemas marinos [5].

Por encontrarse cerca de la costa y del muelle, la presencia cercana de la refinadora de petróleo y el frecuente tránsito de embarcaciones, la isla Uvita es susceptible a la contaminación por hidrocarburos y metales pesados. A lo anterior, se le debe adicionar el posible impacto de las aguas inyectadas por el emisario de la Ciudad de Limón [6].

Acuña y colaboradores [7] evaluaron las concentraciones de metales pesados en varios sitios del país. Las concentraciones más altas de plomo se detectaron en el Caribe y en la Bahía de Golfito, y para los otros elementos los valores máximos se encontraron en la Bahía de Golfito. Se consideró que tanto la Bahía de Moín como el Golfo de Nicoya mostraron una condición intermedia de contaminación. Según los autores, la desembocadura del río Moín es el lugar donde convergen dos fuentes principales de contaminación por hidrocarburos del Caribe costarricense: la Refinadora Costarricense de Petróleo y el muelle.

Otra amenaza para el sitio es el arrastre de sedimentos por parte de los ríos, principalmente del río Cieneguita, los cuales se originan por la deforestación de las montañas tierra adentro, así como por las prácticas agrícolas inapropiadas y las construcciones ubicadas en las zonas costeras; lo cual provoca la afectación de los arrecifes coralinos con sustancias ajenas al mismo, ocasionando un daño al ecosistema marino [7].

El emisario submarino de la ciudad de Limón está dividido en cuatro secciones: a) sección terrestre, b) sección marítima sobre el fondo coralino, c) sección marítima sobre fondo no coralino y d) sección marítima de difusores (comprende el extremo final del emisario submarino, que se extiende sobre formaciones no coralinas) [8].

En el caso particular de la Isla Uvita, debido al sistema de corrientes marinas y la cercanía con la costa, su ecosistema marino se ve influenciado por el manejo de los residuos sólidos y líquidos de la ciudad de Limón, en particular por las aguas residuales descargadas por el emisario submarino [9]. En consecuencia, las aguas negras podrían provocar que los corales sean desplazados por competencia por algas, lo cual modificaría el ecosistema marino de la Isla [9].

\section{Materiales y métodos}

\section{Frecuencia y elección de puntos de muestreo}

El estudio se llevó a cabo en las cercanías de la isla Uvita (figura 2), entre setiembre del 2012 y agosto del 2013. Para la puesta en marcha del proyecto se programaron tres visitas de campo, en función de las épocas climatológicas del año, como se describe a continuación:

- Setiembre 2012 (época lluviosa).

- Octubre 2012 (transición lluviosa-seca).

- Setiembre 2013 (época lluviosa).

Debido a que en la isla Uvita no se presenta una estación seca bien definida; se tomaron las muestras en setiembre, que corresponde al mes más lluvioso en la zona Caribe. Sin embargo, se reconocen dos periodos bien definidos de lluvia: de octubre a enero, y de mayo a agosto [10]. 
La distribución de dichos puntos se aprecia en la figura 3. Los puntos marcados como E1, E2, E3 y E4 se designaron como zonas de influencia por el emisario submarino, mientras que los puntos más alejados representados como E5 y E6 se utilizaron como puntos de control definidos como "zona sin influencia" del emisario; para efectos de la discusión de resultados se hará referencia a las estaciones de muestreo 1, 2, 3, 4, 5 y 6.

En cada punto se recolectaron muestras a dos profundidades, a nivel superficial y a $10 \mathrm{~m}$; los parámetros físicos evaluados en cada punto fueron oxígeno disuelto, temperatura, $\mathrm{pH}$, turbiedad y sólidos. Los análisis químicos realizados fueron nutrientes (nitrógeno amoniacal, nitritos, nitratos, fosfatos y silicatos) y metales pesados ( $\mathrm{Pb}, \mathrm{Ni}$ y $\mathrm{Cr}$ ).

En la literatura consultada no se logró ubicar una norma de evaluación de la calidad de agua de mar; en consecuencia, los datos generados se compararon con los resultados reportados en la literatura para el Golfo de México [11] [12] y la zona del Caribe de Costa Rica [13] [14] . Los valores promedio se analizaron estadísticamente con el programa Minitab 17®.

\section{Resultados y discusión}

Cuadro 1. Valores promedios de las concentraciones ( $\mu \mathrm{mol} / \mathrm{L})$ de nutrientes a nivel superficial y a 10 metros de profundidad.

\begin{tabular}{|c|c|c|c|c|c|c|c|c|c|c|}
\hline $\begin{array}{c}\text { Estación de } \\
\text { muestreo }\end{array}$ & \multicolumn{2}{|c|}{$\mathrm{NO}_{3}^{-}( \pm 0,06)$} & \multicolumn{2}{|c|}{$\mathrm{NO}_{2}^{-}( \pm 0,02)$} & \multicolumn{2}{|c|}{$\mathrm{NH}_{4}^{+}( \pm 0,7)$} & \multicolumn{2}{|c|}{$\mathrm{PO}_{4}^{-3}( \pm 0,16)$} & \multicolumn{2}{|c|}{$\mathrm{SiO}_{4}^{-4}( \pm 0,5)$} \\
\hline Profundidad & $0 \mathrm{~m}$ & $10 \mathrm{~m}$ & $0 \mathrm{~m}$ & $10 \mathrm{~m}$ & $0 \mathrm{~m}$ & $10 \mathrm{~m}$ & $0 \mathrm{~m}$ & $10 \mathrm{~m}$ & $0 \mathrm{~m}$ & $10 \mathrm{~m}$ \\
\hline 1 & 4,46 & 3,05 & 0,68 & 0,97 & 1,8 & 1,0 & 1,02 & 0,33 & 2,0 & 6,4 \\
\hline 2 & 4,86 & 7,48 & 0,25 & 0,56 & 1,0 & 0,6 & 0,83 & 1,13 & 5,5 & 4,2 \\
\hline 3 & 6,19 & 8,19 & 0,23 & 0,33 & 1,6 & 0,3 & 2,75 & 0,62 & 7,7 & 8,3 \\
\hline 4 & 6,00 & 11,54 & 0,28 & 0,24 & 0,8 & 0,2 & 0,43 & 0,49 & 4,0 & 8,7 \\
\hline 5 & 5,32 & 6,93 & 0,28 & 0,36 & 1,3 & 0,4 & 0,65 & 1,40 & 7,9 & 4,8 \\
\hline 6 & 4,65 & 1,87 & 0,20 & 0,73 & 2,2 & 0,7 & 0,54 & 0,76 & 4,8 & 3,9 \\
\hline Promedio & 5,25 & 6,51 & 0,32 & 0,53 & 1,4 & 0,5 & 1,03 & 0,79 & 5,3 & 6,0 \\
\hline $\begin{array}{c}\text { Promedio } \\
\text { General }\end{array}$ & \multicolumn{2}{|c|}{5,88} & \multicolumn{2}{c}{0,43} & & 1,0 & & 0,91 & & 5,7 \\
\hline
\end{tabular}

Los valores promedio (en $\mu \mathrm{mol} / \mathrm{L}$ ) de los nutrientes (nitrato, nitrito, amonio, fósforo y silicio), a nivel superficial y a 10 metros de profundidad, se resumen en el cuadro 1 . Se obtuvieron los siguientes valores promedio: $(5,88 \pm 0,06) \mu \mathrm{mol} / \mathrm{L}$ para $\mathrm{NO}_{3}^{-},(0,43 \pm 0,02) \mu \mathrm{mol} / \mathrm{L}$ para $\mathrm{NO}_{2}^{-},(1,0$ $\pm 0,7) \mu \mathrm{mol} / \mathrm{L}$ para $\mathrm{NH}_{4}^{+} ;(0,91 \pm 0,16) \mu \mathrm{mol} / \mathrm{L}$ para fósforo $\mathrm{P}$ y $(5,7 \pm 0,5) \mu \mathrm{mol} / \mathrm{L}$ para silicio; los datos del cuadro 1 superan en algunos casos los niveles encontrados por Villanueva y Botello [12] en el Golfo de México y por Mora y Portuguez [13] en la zona de influencia de la isla Uvita. Lo anterior sugiere que, si el emisario continúa con las descargas, a mediano o largo plazo podría tener un efecto negativo sobre el ecosistema en los alrededores de la Isla Uvita.

En el cuadro 2 se muestran los valores promedio de los parámetros fisicoquímicos determinados in-situ, los sólidos disueltos y los sólidos sedimentables. Con respecto a los sólidos suspendidos o sedimentables, los niveles constantes en todos los muestreos y épocas del año sugieren que no hay un efecto directo de las descargas de sedimentos a través del emisario submarino 
sobre el entorno circundante; las grandes masas de agua en los mares permiten la dilución y la dispersión homogénea de la materia orgánica, así como de los sólidos suspendidos y sedimentos que pueden llegar a la zona aledaña a la isla por parte del emisario. Los parámetros medidos in situ (temperatura, $\mathrm{pH}$, turbiedad, conductividad y oxígeno disuelto) de la zona de estudio presentaron valores estables y similares entre sí, lo que sugiere que no hay una influencia negativa del emisario submarino sobre estos parámetros en el entorno. Tampoco se observa un cambio significativo de alguno de los parámetros estudiados en función de la estación climatológica.

La comparación de los datos de los cuadros 1 y 2 con la información reportada por Villanueva y Botello ([12] para el Golfo de México y por Mora y Portuguez [13] para las cercanías de la isla Uvita, sugiere que el emisario submarino tiene un impacto ligero en el ecosistema marino; sin embargo, se necesita realizar una mayor cantidad de repeticiones de los parámetros analizados en esta sección para tener un mejor panorama del efecto del emisario en la zona de estudio.

Cuadro 2. Valores promedio de los parámetros determinados in-situ y de los sólidos a nivel superficial y a 10 metros de profundidad, en seis estaciones de muestreo frente a la Isla Uvita, Limón, entre el 2012 y el 2013.

\begin{tabular}{|c|c|c|c|c|c|c|c|c|c|c|c|c|c|}
\hline $\begin{array}{c}\text { Estación de } \\
\text { muestreo }\end{array}$ & \multicolumn{2}{|c|}{$\begin{array}{c}\mathrm{S} . \mathrm{Sed} \mathrm{mL} / \mathrm{L} \\
( \pm 0,01)\end{array}$} & \multicolumn{2}{|c|}{$\begin{array}{c}\mathrm{S} \text {. Disueltos } \\
\mathrm{g} / \mathrm{L}( \pm 0,2)\end{array}$} & \multicolumn{2}{|c|}{$\mathrm{pH}( \pm 0,1)$} & \multicolumn{2}{|c|}{$\begin{array}{c}\text { Temperatura } \\
{ }^{\circ} \mathrm{C}( \pm 0,1)\end{array}$} & \multicolumn{2}{|c|}{$\begin{array}{c}\text { Oxígeno } \\
\text { disuelto } \mathrm{mg} / \mathrm{L} \\
( \pm 0,1)\end{array}$} & \multicolumn{2}{c|}{$\begin{array}{c}\text { Conductividad } \\
\mu \mathrm{S} / \mathrm{cm}( \pm 1)\end{array}$} & $\begin{array}{c}\text { Turbiedad } \\
\mathrm{m}( \pm 0,1)\end{array}$ \\
\hline Profundidad & $0 \mathrm{~m}$ & $10 \mathrm{~m}$ & $0 \mathrm{~m}$ & $10 \mathrm{~m}$ & $0 \mathrm{~m}$ & $10 \mathrm{~m}$ & $0 \mathrm{~m}$ & $10 \mathrm{~m}$ & $0 \mathrm{~m}$ & $10 \mathrm{~m}$ & $0 \mathrm{~m}$ & $10 \mathrm{~m}$ & $\mathrm{NA}$ \\
\hline 1 & 0,10 & 0,23 & 30,6 & 38,5 & 8,4 & 8,2 & 28,5 & 27,7 & 7,3 & 5,5 & 49600 & 53400 & 0,7 \\
\hline 2 & 0,10 & 0,10 & 35,2 & 36,8 & 8,3 & 8,3 & 28,8 & 28,5 & 7,6 & 5,1 & 49500 & 51300 & 0,7 \\
\hline 3 & 0,10 & 0,10 & 34,4 & 37,6 & 8,5 & 8,2 & 29,6 & 29,3 & 7,6 & 5,3 & 50400 & 51600 & 1,0 \\
\hline 4 & 0,10 & 0,10 & 36,0 & 37,9 & 8,6 & 8,2 & 30,1 & 29,9 & 7,7 & 5,6 & 49900 & 52800 & 2,0 \\
\hline 5 & 0,10 & 0,10 & 40,5 & 37,7 & 8,5 & 8,4 & 28,9 & 28,7 & 8,0 & 5,2 & 47700 & 52800 & 0,8 \\
\hline 6 & 0,13 & 0,10 & 36,3 & 38,7 & 8,1 & 8,1 & 28,4 & 29,1 & 8,1 & 5,2 & 48000 & 52800 & 1,0 \\
\hline
\end{tabular}

En el cuadro 3 se muestran las concentraciones promedio (en $\mathrm{mg} / \mathrm{L}$ ) de los niveles de cromo, plomo y níquel, en las seis estaciones de muestreo. Se observó que, en general, las concentraciones de $\mathrm{Ni}^{2+}$ fueron mayores a las de $\mathrm{Pb}^{2+}$ y éstas últimas un poco mayores a las de $\mathrm{Cr}^{3+}$. Los niveles de los metales pesados superaron los valores reportados para el Golfo de México [12]: (0,00002-0,00015 mg/L para cromo; 0,001-1,963 mg/L para plomo; 0,00000050,006 mg/L para níquel). Es importante mencionar que se detectaron niveles muy altos de estas especies en la tercera actividad de muestreo; la presencia de estos metales sugiere que la zona de estudio podría estar siendo influenciada por otras actividades antropogénicas ajenas al emisario submarino de la ciudad de Limón, así como por la constante actividad portuaria y de la industria petrolera cercana a la zona. En ese sentido, se hace necesario realizar nuevos muestreos para identificar la causa principal de la presencia de los metales detectados; adicionalmente, se requiere evaluar los niveles de metales en las aguas que llegan al emisario, actividad que no se pudo llevar a cabo debido a que la institución que lo administra no otorgó el permiso correspondiente. 
Cuadro 2. Promedios de los metales pesados analizados $(\mathrm{Pb}, \mathrm{Cr}$ y $\mathrm{Ni}$ ) a nivel superficial y a 10 metros de profundidad.

\begin{tabular}{|c|c|c|c|c|c|c|}
\hline $\begin{array}{c}\text { Estación de } \\
\text { muestreo }\end{array}$ & \multicolumn{2}{|c|}{$\begin{array}{c}\text { Cromo } \mathrm{mg} / \mathrm{L} \\
( \pm 0,025)\end{array}$} & \multicolumn{2}{|c|}{$\begin{array}{c}\text { Plomo } \mathrm{mg} / \mathrm{L} \\
( \pm 0,025)\end{array}$} & \multicolumn{2}{|c|}{$\begin{array}{c}\text { Níquel } \mathrm{mg} / \mathrm{L} \\
( \pm 0,025)\end{array}$} \\
\hline Profundidad & $0 \mathrm{~m}$ & $10 \mathrm{~m}$ & $0 \mathrm{~m}$ & $10 \mathrm{~m}$ & $0 \mathrm{~m}$ & $10 \mathrm{~m}$ \\
\hline 1 & 0,166 & 0,179 & 0,328 & 0,308 & 0,626 & 0,489 \\
\hline 2 & 0,180 & 0,203 & 0,303 & 0,372 & 0,617 & 0,815 \\
\hline 3 & 0,189 & 0,183 & 0,443 & 0,290 & 0,416 & 0,493 \\
\hline 4 & 0,142 & 0,151 & 0,326 & 0,394 & 0,305 & 0,665 \\
\hline 5 & 0,180 & 0,180 & 0,438 & 0,356 & 0,513 & 0,472 \\
\hline 6 & 0,166 & 0,196 & 0,312 & 0,354 & 0,650 & 0,685 \\
\hline
\end{tabular}

La información del cuadro 3 no permite establecer si el emisario submarino, en lo concerniente a metales pesados, tiene una influencia directa sobre el entorno. Tampoco se detectaron diferencias con el cambio de época lluviosa a seca y viceversa. Tal parece que tanto el efecto de dilución de la masa oceánica como las corrientes marinas, tienden a homogenizar los posibles contaminantes inyectados al océano por parte del emisario submarino; adicionalmente, no se observan comportamientos atípicos con el cambio de estación climatológica.

\section{Conclusiones}

Los datos encontrados en el presente estudio, no permiten afirmar que las descargas del emisario submarino de la ciudad de Limón tienen una influencia significativa sobre el entorno de la Isla Uvita. El aumento en los niveles de metales pesados detectados en la última actividad de muestreo más parece obedecer a factores antropogénicos y naturales de la zona, que no están ligados exclusivamente a las descargas del emisario submarino. No obstante, este último podría aportar contaminantes a mediano y largo plazo al ecosistema marino de la zona.

Se identificaron tres posibles factores de riesgo ambiental: la constante actividad portuaria, el crecimiento de la industria petrolera y el flujo de inyección del emisario submarino; dichas actividades podrían generar desechos potencialmente tóxicos para el entorno marino.

Las estaciones de referencia (5 y 6) mostraron un comportamiento similar a las estaciones de influencia (1, 2, 3 y 4) en la mayoría de parámetros analizados; lo anterior sugiere que hay un efecto directo del emisario y de los demás emisores de contaminantes sobre las estaciones denominadas como zona de referencia (5 y 6); sin embargo, se necesita recolectar más información para definir el impacto del emisario sobre dichos puntos.

\section{Referencias}

[1] C. Gamboa, «Estudio de Impacto Ambiental del Proyecto del Emisario Submarino para el Alcantarillado Sanitario de la Ciudad de Limón. Anexo 4: "Estado actual de las 134 comunidades marinas en los alrededores de la Isla Uvita, Limón, Costa Rica”, » PROIGE, San José, Costa Rica, 1998.

[2] F. Ruiz, «Variables de medición de indicadores de gestión de excretas y aguas residuales,» Focards-APS, San José, Costa Rica, 2012. 
[3] «Decreto No. 30413. Reglamento Sectorial para la Regulación de los Servicios de Acueducto y Alcantarillado Sanitario. Costa Rica.,» La Gaceta No. 91., 2002.

[4] A. Ramírez y E. Salazar, «Calidad de aguas residuales en los sistemas de depuración operados y administrados por AYA y estudios especiales de interés institucional, Instituto Costarricense de Acueductos y Alcantarillados, San José, 2005.

[5] E. Aguilar, M. Ballestero, J. Echeverría y C. \&. O. V. R. Espinosa, «Primera etapa del plan de manejo integral del recurso hídrico: una estrategia nacional para el MIRH., » Ministerio del Ambiente y Energía de Costa Rica (MINAE), San José, Costa Rica, 2004.

[6] J. M. Acuña, «Calidad de aguas residuales en los sistemas de depuración operados y administrados por AyA y estudios especiales de interés institucional. Instituto Costarricense de acueductos y alcantarillados, » Instituto Costarricense de acueductos y alcantarillados, San José, Costa Rica, 2005.

[7] J. García, J. Acuña y J. Vargas, «Metales traza en sedimentos costeros de Costa Rica, Revista de Biología Tropical, vol. 52, pp. 51-60, 2004.

[8] H. Salas, «Emisarios submarinos: Enfoque general, conceptos básicos de diseño y requerimientos de datos para América Latina y el Caribe,» CEPIS, 1998.

[9] C. Pérez, Interpretación ambiental de un sendero autoguiado en Isla Quiribrí de Puerto Limón, como un aporte al desarrollo turístico de la Región Caribe de Costa Rica., San José: Universidad de Costa Rica, 2003.

[10] I. M. N. (IMN), Comité Nacional de Hidrología y Meteorología de Costa Rica (CHRM Costa Rica), Balance hídrico superficial de Costa Rica, PHI-LAC., documento técnico No 10, UNESCO, 2003.

[11] F. Contreras, O. Castañeda, R. Torres-Alvarado y F. Gutiérrez, «Nutrientes en 39 lagunas costeras mexicanas, » Revista de Biología Tropical, vol. 44, n² 2, pp. 417-425, 1996.

[12] S. F. Villanueva y A. V. Botello, «Metales pesados en la zona costera del Golfo de México y Caribe Mexicano: una revisión., » Revista internacional de contaminación ambiental, vol. 8, nº 1, pp. 47-61, 1992.

[13] D. Mora-Alvarado y C. Portuguez-Barquero, «Cobertura de la disposición de excretas en Costa Rica para el periodo 2000-2014 y expectativas en el 2021, „Tecnología en Marcha, vol. 29, n² 2, pp. 43-62, 2016.

[14] INVEMAR, «Diagnóstico y evaluación de la calidad ambiental marina en el Caribe y Pacífico colombiano. Red de vigilancia para la conservación y protección de las aguas marinas y costeras de Colombia. Diagnóstico nacional y regional., » 2005. 